\title{
Commentary: Expanding the salvage time window for large-vessel occlusion stroke after cardiovascular surgery
}

\author{
Suk Jung Choo, MD, PhD
}

\footnotetext{
From the Department of Thoracic and Cardiovascular Surgery, University of Ulsan College of Medicine, Asan Medical Center, Seoul, Korea.

Disclosures: Author has nothing to disclose with regard to commercial support.

Received for publication Jan 8, 2019; accepted for publication Jan 9, 2019; available ahead of print Feb 20, 2019.

Address for reprints: Suk Jung Choo, MD, PhD, Department of Thoracic and Cardiovascular Surgery, Asan Med-

ical Center, University of Ulsan College of Medicine, 88 Olympic-ro 43-gil, Songpa-gu, Seoul, 05505, Korea

(E-mail: sjchoo@amc.seoul.kr)

J Thorac Cardiovasc Surg 2019;158:197-8

$0022-5223 / \$ 36.00$

Copyright (C) 2019 Published by Elsevier Inc. on behalf of The American Association for Thoracic Surgery

https://doi.org/10.1016/j.jtcvs.2019.01.033
}

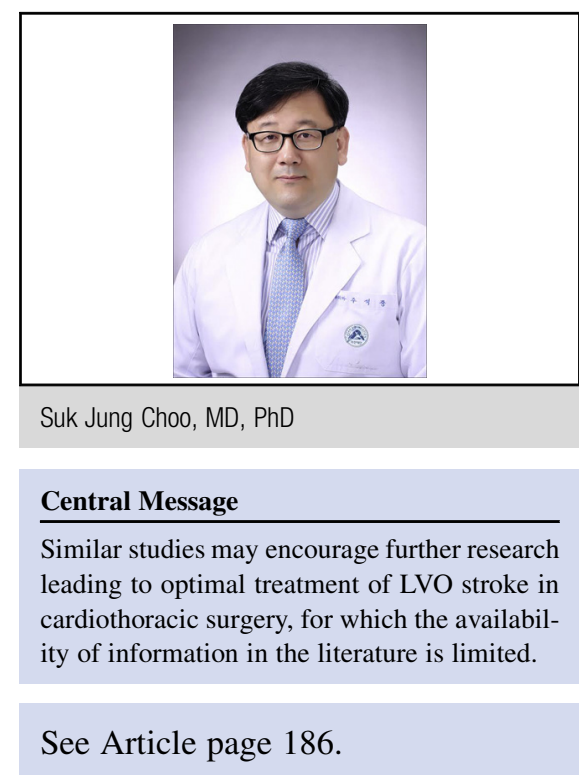

In this issue of the Journal, Sheriff and colleagues ${ }^{1}$ report their study that investigated the incidence of large-vessel occlusion (LVO) stroke after cardiovascular surgery and explored the feasibility of expanding the salvageable time window beyond the currently perceived time limit for reversing neurologic deficits. Timely restoration of circulatory perfusion before the establishment of irreversible damage is of the essence in any organ ischemia. With major LVO stroke, partial or complete reversal of neurologic deficit through intracranial thrombectomy when possible has generally been believed to be achievable when performed within 6 hours of onset, ${ }^{2}$ but additional benefits were believed to diminish beyond this period. ${ }^{3}$ To date, studies investigating the role of thrombectomy for largevessel stroke after cardiovascular surgery have been limited, and there have also been difficulties with accurately determining the duration between the last seen well period to detection. The recently published Clinical Mismatch in the Triage of Wake Up and Late Presenting Strokes Undergoing Neurointervention With Trevo (DAWN) ${ }^{4}$ and Endovascular Therapy Following Imaging Evaluation for Ischemic Stroke 3 (DEFUSE 3$)^{5}$ trials showed that neurologic outcomes were better after thrombectomy than after standard medical treatment in patients with LVO stroke in whom the duration from the last seen well period was greater than 6 hours but complete infarction had not yet set in. Sheriff and colleagues ${ }^{1}$ reviewed their institutional cardiothoracic surgery database during a 5 -year period in which LVO stroke was observed in 15 out of 137 ischemic stroke patients among a total cohort of 5947 cardiovascular surgery patients. Of the $15 \mathrm{LVO}$ patients, 7 patients who were deemed candidates for emergent thrombectomy formed the basis of this report. Although LVO stroke is a relatively rare subtype of ischemic stroke, its significance lies in comprising the majority of stroke-related deaths and severe disabilities. ${ }^{4,5}$ In the study of Sheriff and colleagues, ${ }^{1}$ the overall incidence of postoperative stroke was relatively rare at $2.48 \%$, and most of the strokes after cardiovascular surgery were ischemic in origin, which accounted for $92.5 \%$ of the stroke patients, with LVO accounting for $10.9 \%$ of the strokes. These are the types of stroke that may be amenable to treatment by intervention aimed at removing thrombus, fat, or other solid materials, with expectations of significant improvement if infarction has not yet set in. To this end, a strong and dedicated stroke team with established protocols for rapid intervention is an essential element that may reduce the latency to treatment.

According to Sheriff and colleagues, ${ }^{1}$ LVO stroke after cardiovascular surgery may fall into 2 categories: "wakeup" stroke, which is detected on emergence from anesthesia, and "awake" stroke, in which neurologic deficit occurs after a period of an awake period. From a diagnostic standpoint, the patients with wake-up stroke pose the greatest challenge, because it is difficult to pinpoint the stroke onset once anesthesia induction has been completed. Considering that the majority of cerebrovascular events associated with cardiothoracic surgery are macroembolic in nature, the stroke onset may be blamed on any of the possible causative maneuvers and manipulations, such as clamp placement or removal, occurring at any time after anesthesia. The estimated ischemic duration may therefore depend on when the specific culprit event is considered to have occurred. Although sudden changes in the 
intraoperative monitoring, such as electroencephalographic activity may alert the surgeon to the possibility of an acute adverse event, it may not be possible accurately to pinpoint a neurologic event onset, especially if there was a gradual decrease in electroencephalographic activity before a prolonged aortic crossclamp time, after which further hints of neurologic events may be even more difficult to decipher because the electroencephalographic wave activity may show significantly delayed recovery during the rewarming period, especially if it was combined with a period of hypothermic circulatory arrest. In addition, the normal wake-up period from anesthesia after weaning from a complex cardiothoracic surgery may be prolonged, resulting in inadvertent delay in the detection of neurologic deficit by the usual waiting period for postoperative neurologic recovery. These and other issues, which may all confound determination of the ischemic duration, warrant implementation of measures to spur earlier arousal and acquisition of imaging studies in patients at risk. The logistics of safely transferring these patients to the brain or magnetic resonance imaging scanning facility, let alone incurring additional travel and time away from the intensive care unit to undergo thrombectomy, may be prohibitive, given the typically heavy dependence on invasive treatments such as left ventricular assist devices amidst a complex web of medication lines. At the institution of Sheriff and colleagues, ${ }^{1}$ an immediate head and neck computed tomographic angiogram with delays is acquired in coordination with radiology as a proactive strategy in patients deemed at high risk of LVO during transport from the operating room to the intensive care unit, while the patient is under the care of the anesthetist. These details highlight the necessity of a highly dedicated and coordinated multidisciplinary team approach to overcome the daunting challenges of dealing with the multiple issues facing these patients. In the patients with awake stroke, 5 total in the study of Sheriff and colleagues, ${ }^{1}$ the reaction and ischemic times were relatively quicker, with the time from last seen well to detection ranging from 10 to 150 minutes. Because these patients' conditions were detected in the hospital setting, they were better positioned to benefit from rapid implementation of interventional measures and timely treatment. Nevertheless, the efficient treatment of these patients in general would not be possible without a highly dedicated and efficient stroke team standing by.

For those patients in whom thrombectomy was possible, the overall neurologic outcomes tended to be better than in those patients who did not undergo thrombectomy. Although the study of Sheriff and colleagues ${ }^{1}$ was preliminary in nature because of the small case volume, it nevertheless provided a glimpse into the possibility of treating patients with LVO stroke that would otherwise have been regarded as being too late for treatment or, in the case of wake-up strokes, more or less untreatable. Sheriff and colleagues ${ }^{1}$ have shown that an aggressive strategy to treat these patients at the earliest time possible by a dedicated and tenacious stroke team may result in significantly enhanced neurologic outcomes. Taking the lead from studies such as this, further studies with larger cohorts may be helpful in developing strategies that may better identify patients at risk, as well as in determining the onset and duration of stroke, so that patients in these situations can be treated more effectively and proactively before the establishment of permanent neurologic deficit.

\section{References}

1. Sheriff F, Hisch JA, Shelton K, D'Allesandro D, Stapleton C, Koch M, et al. Large-vessel occlusion stroke after cardiothoracic surgery: expanding time windows offer new salvage opportunities. J Thorac Cardiovasc Surg. 2019;158: 186-96.e2.

2. Goyal M, Menon BK, van Zwam WH, Dippel DW, Mitchell PJ, Demchuk AM, et al; HERMES collaborators. Endovascular thrombectomy after large-vessel ischaemic stroke: a metaanalysis of individual patient data from five randomised trials. Lancet. 2016;387:1723-31.

3. Saver JL, Goyal M, van der Lugt A, Menon BK, Majoie CB, Dippel DW, et al; HERMES Collaborators. Time to treatment with endovascular thrombectomy and outcomes from ischemic stroke: a meta-analysis. JAMA. 2016;316: 1279-88.

4. Nogueira RG, Jadhav AP, Haussen DC, Bonafe A, Budzik RF, Bhuva P, et al; DAWN Trial Investigators. Thrombectomy 6 to 24 hours after stroke with a mismatch between deficit and infarct. N Engl J Med. 2018;378:11-21.

5. Albers GW, Marks MP, Kemp S, Christensen S, Tsai JP, Ortega-Gutierrez S, et al; DEFUSE 3 Investigators. Thrombectomy for stroke at 6 to 16 hours with selection by perfusion imaging. $N$ Engl J Med. 2018;378:708-18. 